\title{
Rhythmical Massage Therapy in Chronic Disease: A 4-Year Prospective Cohort Study
}

\author{
HARALD J. HAMRE, M.D., ${ }^{1}$ CLAUDIA M. WITT, M.D., ${ }^{2}$ \\ ANJA GLOCKMANN, Dipl.Biol., ${ }^{1}$ RENATUS ZIEGLER, Ph.D., ${ }^{3}$ \\ STEFAN N. WILLICH, M.D., M.P.H., ${ }^{2}$ and HELMUT KIENE, M.D. ${ }^{1}$
}

\begin{abstract}
Objective: Rhythmical massage therapy is used in 24 countries but has not yet been studied in outpatient settings. The objective was to study clinical outcomes in patients receiving rhythmical massage therapy for chronic diseases.

Design: Prospective 4-year cohort study.

Setting: Thirty-six (36) medical practices in Germany.

Participants: Eighty-five (85) outpatients referred to rhythmical massage therapy.

Outcome measures: Disease and Symptom Scores (physicians' and patients' assessment, respectively, 0-10) and SF-36. Disease Score was measured after 6 and 12 months, and other outcomes after 3, 6, 12, 18, 24, and 48 months.

Results: Most common indications were musculoskeletal diseases (45\% of patients; primarily back and neck pain) and mental disorders (18\%, primarily depression and fatigue). Median disease duration at baseline was 2.0 years (interquartile range $0.5-6.0$ ). Median number of rhythmical massage therapy sessions was 12 (interquartile range 9-12), and median therapy duration was 84 (49-119) days. All outcomes improved significantly between baseline and all subsequent follow-ups. From baseline to 12 months, Disease Score improved from (mean \pm standard deviation) $6.30 \pm 2.01$ to $2.77 \pm 1.97(p<0.001)$, Symptom Score improved from $5.76 \pm 1.81$ to $3.13 \pm 2.20(p<0.001)$, SF-36 Physical Component score improved from $39.55 \pm 9.91$ to $45.17 \pm 9.88(p<0.001)$, and SF-36 Mental Component score improved from $39.27 \pm 13.61$ to $43.78 \pm 12.32$ $(p=0.028)$. All these improvements were maintained until the last follow-up. Adverse reactions to rhythmical massage therapy occurred in $4(5 \%)$ patients; 2 patients stopped therapy because of adverse reactions.

Conclusions: Patients receiving rhythmical massage therapy had long-term reduction of chronic disease symptoms and improvement of quality of life.

\section{INTRODUCTION}

A nthroposophic medicine (AM) was founded by Rudolf Steiner, Ph.D., Free Academy of Spiritual Science, Dornach, Switzerland, and Ita Wegman, M.D., Free Academy of Spiritual Science, and Clinical Therapeutic Institute, Arlesheim, Switzerland. ${ }^{1}$ AM extends conventional medicine through a holistic approach to humans and nature, addressing physical,

psychologic, and spiritual aspects of the patients (Table 1). ${ }^{2,3}$ AM is provided by physicians (counseling, AM medication) and nonmedical therapists (art therapy, eurythmy movement exercises, and rhythmical massage therapy [RMT]). ${ }^{2}$

RMT was developed from Swedish massage by Dr. Wegman, a physician and physiotherapist. ${ }^{4}$ Special RMT techniques include lifting movements, rhythmically undulating gliding movements, and complex movement patterns such
\end{abstract}

\footnotetext{
${ }^{1}$ Institute for Applied Epistemology and Medical Methodology, Freiburg, Germany.

${ }^{2}$ Institute for Social Medicine, Epidemiology, and Health Economics, Charité University Medical Center, Berlin, Germany.

${ }^{3}$ Society for Cancer Research, Arlesheim, Switzerland.
} 
Table 1. Description of Rhythmical Massage Therapy (RMT)

Postulated
working principles

Indications

Contraindications

Overall structure

of therapy

Use of conversation

Use of music

Lubricants

Body areas

massaged

Supplementary
organ treatment
Massage
techniques
Movement
direction
Special movement
patterns

Temperature

Rest period after massage

Duration of treatment

Frequency of therapy sessions

Number of sessions

Supplementary advice and information
According to the anthroposphic understanding of humans and nature, four different classes of formative forces can be discerned: (1) in minerals, material forces of physico-chemical matter; (2) in plants, formative vegetative forces interact with material forces, bringing about and maintaining the living form; (3) in animals with sensory and motor systems and with a corresponding inner life, a further class of formative forces (anima, soul) interacts with material and vegetative forces; (4) in the human organism with its individual mind and capacity of thinking, another class of formative forces (Geist, spirit) interacts with the material, vegetative, and mineral forces. The interactions of these forces are understood to vary between different regions and organs in the human body, resulting in a complex equilibrium. This equilibrium can be distorted in various forms of human disease, and is sought to be regulated by RMT and other anthroposophic therapies.

RMT is used for a wide range of indications in family practice, internal medicine, surgery, orthopedics, neurology, gynecology, pediatrics, geriatrics, psychiatry, and rehabilitation.

Cancer (nonremitted or not radically operated), acute infections, acute eczema, pregnancy.

First 1-2 sessions are primarily diagnostic, gathering verbal, visual, and tactile information from the patient: body proportions and posture, quality of skin and soft tissues, muscle tone, warmth distribution, respiration, sensitivity to touch. Remaining sessions are primarily therapeutic.

First 1-2 sessions: extensive. Remaining sessions: initial brief conversation only.

No.

Vegetable oils, used sparingly.

In general, RMT of the arms or upper back aims to intensify the involvement of soul and spirit forces with material and vegetative forces, whereas massage of the lower limbs aims to weaken this involvement. Therapists may also work on one part of the body to create an effect in another part. Special massage programs (each with specified patient position, sequence of techniques, movement patterns, and duration of treatment) exist for the hands, arms, anterior and posterior lower limbs, feet, knees, hips, lower back, whole back, neck, abdomen, and head.

Optionally, an ointment containing metals such as copper or iron is lightly rubbed into the skin near a major organ (liver, kidneys, heart, or spleen) to stimulate, calm, or harmonize organ function. Duration of treatment $1-2$ min.

Effeurage (gliding) with light, rhythmically undulating pressure, keeping therapist's palm in good contact with patient's skin. Kneading with circular, loop-shaped movements. Gentle lifting movements with both hands. Friction. Percussion. Vibration.

Differentiation: e.g., massaging the legs upwards can create a greater sense of vitality and "awakeness"; working with the legs downwards is used to "anchor" patients back into their bodies.

Circle: single or double, i.e., performed with reciprocal phase-displaced movements of both hands. Spiral: single (e.g., for abdomen, liver, knee, or knuckle) or moving upward (e.g., posterior thigh, upper back). Spiral + circle (soothing heart treatment). Lemniscate = figure of eight: fixed (stimulating heart treatment) or moving upwards (whole back). Lemniscate forms can be symmetric (lower ack), have different size of the two circles (spleen), or asymmetric crossing of the circles (kidney). Zig-zag: downward (abdomen). Centripetal (lower back, forehead).

Throughout the massage the patient is kept warm, with only the massaged parts of the body exposed. Windows are closed; draughts are avoided. Mild hot packs may be applied.

Considered essential to achieve desired effects. The patient is wrapped in towels and blankets and left in a darkened room.

Massage: usually 20-30 min. Rest period: at least $20 \mathrm{~min}$.

Once or twice weekly.

Usually 6-12 sessions.

Lifestyle changes (e.g. diet, alcohol, or smoking restriction, exercises), footbaths or limb washes with particular oils may be recommended.

Table structure adapted from Goldstone LA. Massage as an orthodox medical treatment past and future. Complement Ther Nurs Midwifery 2000;6:169-175. Description condensed from refs. 1-4. Also from Glöckler M, Schürholz J, Treichler M. Anthroposophic Medicine [in German]. In: Zentrum zur Dokumentation für Naturheilverfahren e.V., Forschungsinstitut Freie Berufe, eds. Documentation of Special and Naturopathic Therapy Systems in Europe [in German]. Essen: VGM-Verlag, 1991:214-335.

as lemniscates (Table 1). In addition to effects on the skin, subcutaneous tissues, and muscles, RMT is believed to have both general effects (e.g., enhancing physical vitality) and disease-specific effects (Table 1). RMT can be used as monotherapy or in conjunction with other therapies. RMT is practiced by physiotherapists with additional $1 \frac{1}{1} 2-3$ years of RMT training according to a standardized curriculum. RMT schools exist in Australia, Germany, Italy, South 
Africa, Switzerland, and the United States. Presently, RMT is provided by approximately 700 therapists in 24 countries worldwide (U. Niedermann, International Coordination AM, personal communication, May 2007).

Observational studies in inpatient settings suggest that RMT can have clinically relevant effects. ${ }^{3,5,6}$ However, these studies evaluated several AM therapies, and only a proportion of the patients received RMT. Here we present a long-term study of RMT users.

\section{MATERIALS AND METHODS}

\section{Study design and objective}

This prospective cohort study was part of a research project on the effectiveness and costs of AM therapies in outpatients with chronic disease (Anthroposophic Medicine Outcomes Study, AMOS). ${ }^{7,8}$ The AMOS project was initiated by a health insurance company in conjunction with a health benefit program. The present study assessed symptoms, quality of life, adjunctive therapies, adverse reactions, and therapy satisfaction in outpatients receiving RMT under routine clinical conditions.

\section{Setting and participants}

All physicians certified by the Physicians' Association for Anthroposophical Medicine in Germany and treating outpatients in Germany were invited to participate. The participating physicians were instructed to recruit consecutive outpatients ages 1-75, referred to RMT for any indication (main diagnosis). Exclusion criteria were previous RMT for main diagnosis, or ongoing RMT. RMT therapists were certified by the German Rhythmical Massage Therapy Association. RMT was implemented according to the RMT therapists' discretion. Other therapies such as AM medication, AM art or eurythmy exercises, or non-AM adjunctive therapies were allowed throughout the study.

Participating physicians $(n=36)$ did not differ significantly from all AM-certified physicians in Germany $(\mathrm{n}=$ 362 ) regarding gender (56\% versus $62 \%$ males), age (mean \pm standard deviation $47.3 \pm 7.2$ versus $47.5 \pm 7.9$ years), number of years in practice $(19.3 \pm 7.6$ versus $19.5 \pm 8.7)$, and the proportion of primary care physicians (94\% versus $85 \%$ ). Participating therapists $(n=33)$ did not differ from RMT therapists without study patients $(\mathrm{n}=122)$ regarding gender (76\% versus $74 \%$ females), age (mean $50.1 \pm 7.2$ versus $50.0 \pm 9.7$ years), or number of years since RMT certification (mean $7.7 \pm 2.0$ versus $7.4 \pm 3.7$ years).

\section{Clinical outcomes}

Since the study encompassed different diagnoses, disease severity was assessed uniformly for all diagnoses on numerical scales from 0 ("not present") to 10 ("worst possible"):
Disease Score (physician's assessment of severity of main diagnosis); Symptom Score (patients' assessment of one to six most relevant symptoms present at baseline). In patients $\geq 17$ years, quality of life was assessed with a widely used generic instrument, the SF (Short Form)-36 Health Survey: Physical and Mental Component Summary Measures, the eight SF-36 scales, and the SF-36 Health Change item. ${ }^{9}$ Disease Score was documented after 0, 6, and 12 months, Symptom Score and SF-36 after $0,3,6,12,18,24$, and 48 months.

\section{Other outcomes}

- Adjunctive therapy use

- Therapy ratings

- Adverse reactions (physician and patient documentation).

\section{Data collection}

All data were documented with questionnaires sent in sealed envelopes to the study office. Physicians documented eligibility criteria; therapists documented RMT administration; remaining items were documented by patients/caregivers, unless otherwise stated. Patient responses were not made available to physicians. Physicians were compensated

40 Euro (US \$55) per patient, patients received no compensation.

\section{Quality assurance and adherence to regulations}

The study was approved by the Ethics Committee of the Faculty of Medicine Charité, Humboldt University Berlin, and was conducted according to the Helsinki Declaration and the International Conference on Harmonisation-Good Clinical Practice Guidelines. Written informed consent was obtained from all patients before enrollment.

\section{Data analysis}

Data analysis (SPSS ${ }^{\circledR}$ 13.0.1 [SPSS, Inc., Chicago, IL] and StatXact ${ }^{\circledR}$ 5.0.3 [Cytel Software Corporation, Cambridge, $\mathrm{MA}])$ was performed on all patients fulfilling eligibility criteria. The two-tailed $t$-test was used for continuous data, twotailed McNemar test and Fisher's exact test for dichotomous data. Significance criteria were $p<0.05$ and $95 \%$ confidence interval not including 0 . Because this was a descriptive study, no adjustment for multiple comparisons was performed. ${ }^{10}$ Pre-post effect sizes were calculated as Standardized Response Mean and classified as small (0.20-0.49), medium $(0.50-0.79)$, and large $(\geq 0.80){ }^{11}$

\section{RESULTS}

\section{Patient recruitment and follow-up}

From July 1, 1998 to March 31, 2001, a total of 85 patients were included in the study (Fig. 1). The last patient follow-up ensued March 30, 2005. Ninety-six percent (96\%) 


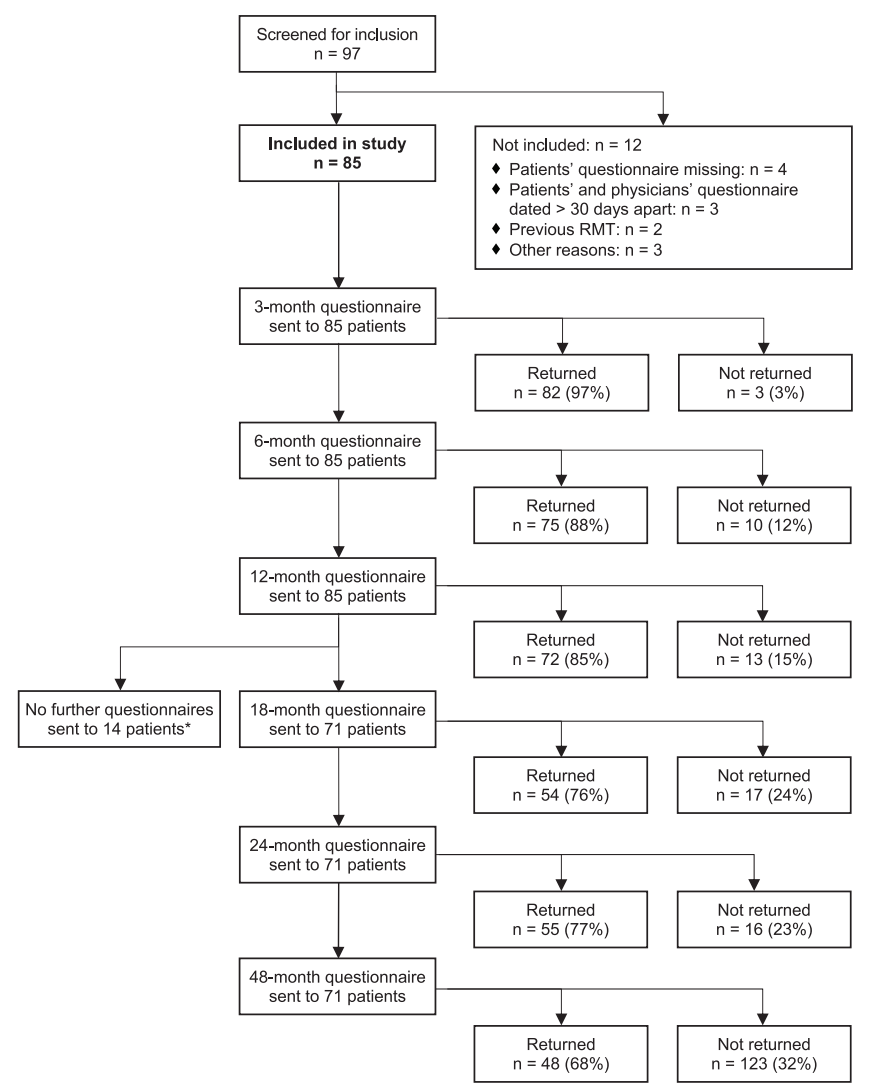

FIG. 1. Patient recruitment and follow-up. *18-, 24-, and 48month follow-up questionnaires were not sent to patients enrolled before January 1, 1999.

(82/85) of patients returned at least one follow-up questionnaire. Respondents and nonrespondents of the 12-month questionnaire did not differ significantly regarding gender, main diagnosis, duration of main diagnosis, baseline Disease Score, or baseline Symptom Score. Median age was 19.0 (interquartile range [IQR] 8.0-37.5) years in nonrespondents and $37.5(29.5-50.8)$ years in respondents $(p=0.009)$.

The total number of patients referred to RMT during the study was estimated by the physicians (response rate $64 \%$, $23 / 36$ physicians). The proportion of referred versus enrolled patients was median 6.6 (IQR 1.8-12.4). There was no sig- nificant correlation between this proportion and the 0-12month improvement of Symptom Score (Spearman-Rho $0.10, p=0.524, \mathrm{n}=44$ patients).

\section{Baseline characteristics}

Patients were recruited from 12 of 16 German federal states. Median age was 37.0 years (IQR 20.5-48.0, mean $35.7 \pm 19.3$ years), and $76 \%(65 / 85)$ of patients were women. Physicians' setting was primary care practice $(95 \%$ of patients, $\mathrm{n}=81 / 85)$, referral practice $(1 \%)$, and outpatient clinic (4\%).

Most frequent main diagnoses, classified by ICD-10 (International Classification of Diseases, Tenth Edition), were M00-M99 Musculoskeletal Diseases (45\%, n = 38/85 patients), F00-F99 Mental Disorders (18\%), and G00-G99 Nervous System Disorders (8\%). Most frequent single diagnoses were back and neck pain (32\%, $\mathrm{n}=27 / 85$ patients), depression/fatigue $(8 \%)$, headache/migraine $(7 \%)$, and fibromyalgia $(5 \%)$. Median duration of main diagnosis was 2.0 years (IQR 0.5-6.0, mean $5.2 \pm 7.8$ years).

Patients had median 2.0 (IQR 1.0-3.0) comorbid diseases. Most common comorbid diseases were F00-F99 Mental Disorders (20\%, 27/137 diagnoses), M00-M99 Musculoskeletal Diseases (15\%), and I00-I99 Circulatory Diseases (12\%).

\section{Therapies}

RMT administration was documented during the first 24 months after study enrollment. In this period 91\% (77/85) of patients had RMT; for 9\% RMT documentation is incomplete. RMT started a median of 7 (IQR 0-18) days after enrollment. Median therapy duration was 84 (IQR 49-119) days, and the median number of RMT sessions was 12 (9-12). During the first 6 study months, 76\% (65/85) of patients used AM medication, 14\% (12/85) had AM eurythmy therapy, and $2 \%(2 / 85)$ had AM art therapy.

Use of diagnosis-related adjunctive therapies within the first 6 study months was analyzed in patients with a main diagnosis of musculoskeletal or mental diseases or headache disorders $(\mathrm{n}=59$, Table 2$)$. Of 51 evaluable patients, $47 \%$ $(n=24)$ had no diagnosis-related adjunctive therapy.

Table 2. Use of Diagnosis-Related Adjunctive Therapies Within the First 6 Study Months

\begin{tabular}{|c|c|c|c|}
\hline $\begin{array}{l}\text { Main diagnosis } \\
(I C D-10)\end{array}$ & Diagnosis-related therapies & $\begin{array}{l}\text { Patients } \\
\text { (n) }\end{array}$ & $\begin{array}{l}\text { Patients without } \\
\text { therapy }(\%)\end{array}$ \\
\hline $\begin{array}{l}\text { Musculoskeletal } \\
\text { diseases (M00-M99) }\end{array}$ & $\begin{array}{l}\text { Immunosuppressive, musculoskeletal, analgesic and } \\
\text { antidepressant drugs, physiotherapy or relevant surgery }\end{array}$ & 35 & $14(40 \%)$ \\
\hline $\begin{array}{l}\text { Mental diseases } \\
\quad(\text { F00-F99) }\end{array}$ & $\begin{array}{l}\text { Psychotherapy (in children ergotherapy or play therapy), } \\
\text { antiepileptic, psycholeptic, analeptic, and anti-addiction drugs }\end{array}$ & 11 & $7(64 \%)$ \\
\hline $\begin{array}{l}\text { Headache disorders } \\
\text { (G43-G44, R51) }\end{array}$ & Analgesics, antimigraine drugs, and antidepressants & 5 & $3(60 \%)$ \\
\hline Total & & 51 & $24(47 \%)$ \\
\hline
\end{tabular}

ICD-10, International Classification of Diseases, Tenth Edition. 

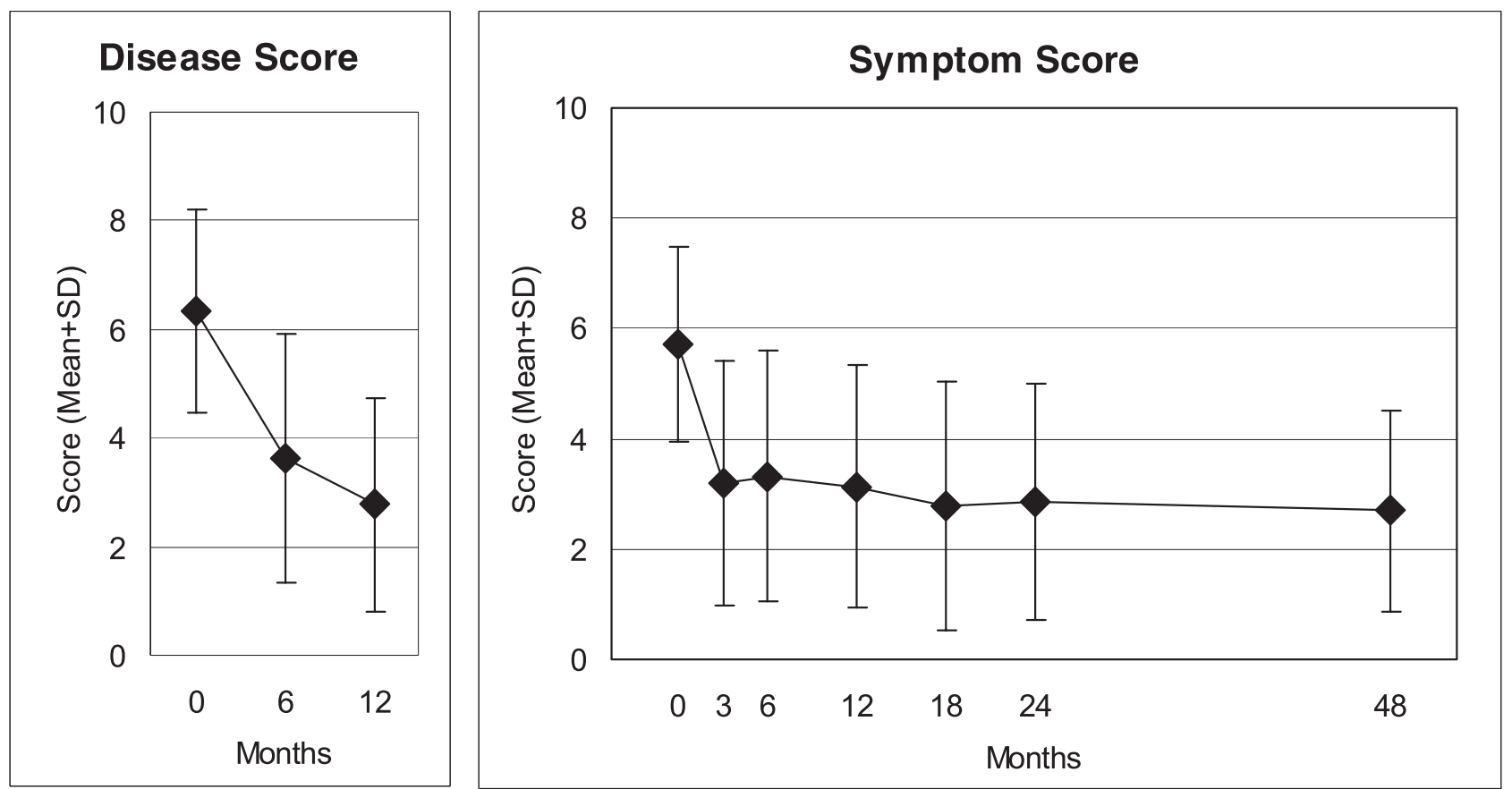

FIG. 2. Disease Score (physicians' assessment), Symptom Score (patients' assessment), 0 “not present," 10 “worst possible.”

\section{Clinical outcomes}

Disease and Symptom Scores (Fig. 2) and the 11 SF-36 scores (Fig. 3) improved significantly between baseline and most subsequent follow-ups (69 significant and 5 nonsignificant improvements in 74 pre-post comparisons). Most improvements occurred during the first 6 months. After 12 months, Disease and Symptom scores were improved from baseline in $89 \%$ and $85 \%$ of patients, respectively (Table 3 ); an improvement of

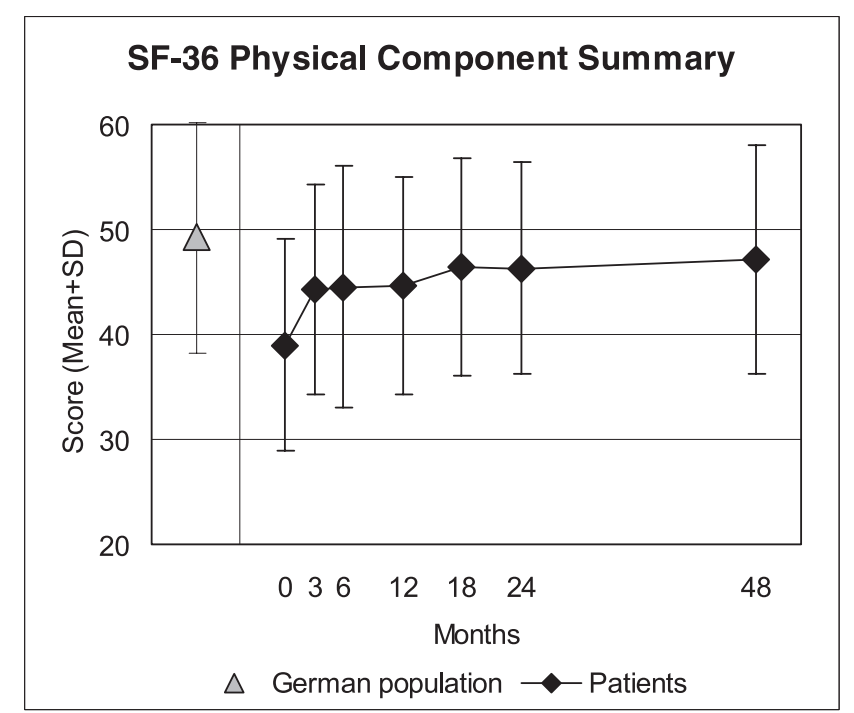

$\geq 30 \%$ of baseline scores was observed in $82 \%$ (46/56) and 64\% (38/59) of evaluable patients, respectively. Disease and Symptom Scores improved similarly in adults and in children. Effect sizes for the 0-12-month comparison were large for Disease and Symptom Scores (1.45 and 1.14) and small-to-medium (range 0.33-0.63) for SF-36 scores. All these improvements were maintained until the last follow-up.

In order to test the influence of four bias factors on 0-12month Symptom Score outcomes, we performed post hoc

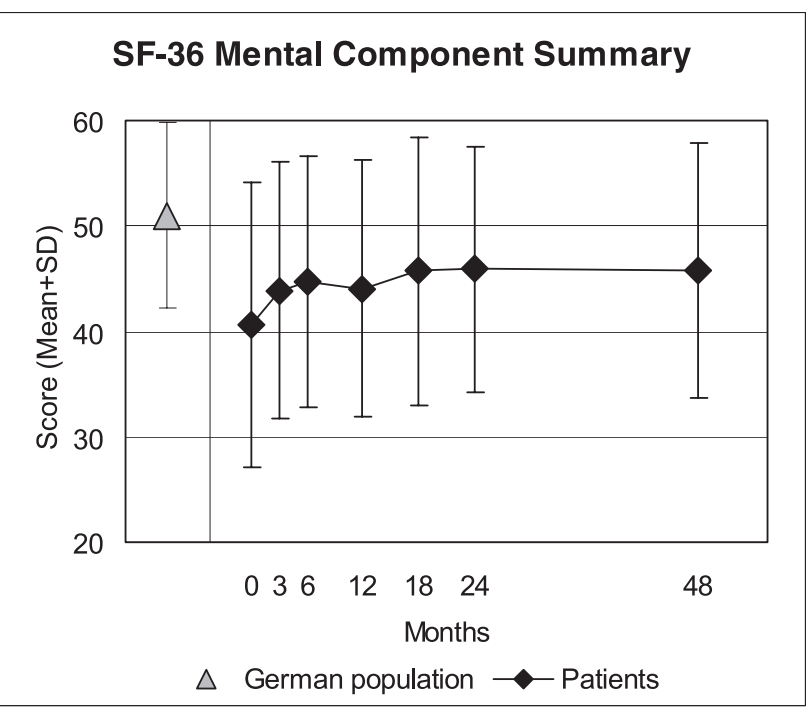

FIG. 3. SF (Short Form)-36 Physical and Mental Component Summary Measures. Higher scores indicate better health. Adult patients and German population (age 17-74 years). ${ }^{9}$ 
Table 3. Clinical Outcomes 0-12 Months

\begin{tabular}{|c|c|c|c|c|c|c|c|}
\hline \multirow[b]{2}{*}{ Item } & \multirow[b]{2}{*}{$N$} & \multirow{2}{*}{$\frac{O \text { months }}{\text { Mean } \pm S D}$} & \multirow{2}{*}{$\frac{12 \text { months }}{\text { Mean } \pm S D}$} & \multirow[b]{2}{*}{$\mathrm{p}$ value } & \multirow{2}{*}{$\frac{0-12-\text { month difference }}{\text { Mean }(95 \% C I)}$} & \multirow{2}{*}{$\begin{array}{c}\text { Improved } \\
(\%)\end{array}$} & \multirow[b]{2}{*}{$S R M$} \\
\hline & & & & & & & \\
\hline Disease score $(0-10)$ & 56 & $6.30 \pm 2.01$ & $2.77 \pm 1.97$ & $p<0.001$ & $3.54(2.88-4.19)$ & 89 & 1.45 \\
\hline Symptom score $(0-10)$ & 59 & $5.76 \pm 1.81$ & $3.13 \pm 2.20$ & $p<0.001$ & $2.63(2.02-3.23)$ & 85 & 1.14 \\
\hline \multicolumn{8}{|l|}{ SF-36 scales $(0-100)$} \\
\hline Physical function & 58 & $71.93 \pm 23.78$ & $78.97 \pm 23.43$ & $p=0.014$ & 7.03 (1.45-12.61) & 57 & 0.33 \\
\hline Role Physical & 57 & $38.16 \pm 36.93$ & $62.72 \pm 40.95$ & $p<0.001$ & $24.56(13.46-35.67)$ & 51 & 0.59 \\
\hline Role Emotional & 54 & $48.15 \pm 42.79$ & $66.05 \pm 42.70$ & $p=0.003$ & $17.90(6.22-29.59)$ & 39 & 0.42 \\
\hline Social Functioning & 58 & $62.93 \pm 25.86$ & $75.00 \pm 24.33$ & $p=0.001$ & $12.07(4.86-19.27)$ & 55 & 0.44 \\
\hline Mental Health & 57 & $55.16 \pm 20.59$ & $61.96 \pm 19.27$ & $p=0.003$ & $6.81(2.40-11.22)$ & 63 & 0.41 \\
\hline Bodily Pain & 58 & $43.10 \pm 23.37$ & $60.66 \pm 29.17$ & $p<0.001$ & $17.55(10.28-24.82)$ & 76 & 0.63 \\
\hline Vitality & 57 & $38.27 \pm 19.45$ & $48.86 \pm 20.96$ & $p<0.001$ & $10.59(5.42-15.75)$ & 63 & 0.54 \\
\hline General Health & 58 & $49.15 \pm 20.28$ & $56.59 \pm 23.37$ & $p=0.001$ & $7.45(3.38-11.51)$ & 62 & 0.48 \\
\hline$S F-36$ Health Change $\left(1-5^{\mathrm{b}}\right)$ & 58 & $3.29 \pm 1.14$ & $2.29 \pm 1.09$ & $p=0.001$ & $0.38(0.17-0.59)$ & 59 & 0.57 \\
\hline SF-36 Physical Component & 54 & $39.55 \pm 9.91$ & $45.17 \pm 9.88$ & $p<0.001$ & $5.62(2.94-8.29)$ & 76 & 0.57 \\
\hline SF-36 Mental Component & 54 & $39.27 \pm 13.61$ & $43.78 \pm 12.32$ & $p=0.001$ & $4.51(1.12-7.90)$ & 57 & 0.36 \\
\hline
\end{tabular}

aPositive differences indicate improvement.

${ }^{\mathrm{b}} 1$ = "much better now than one year ago," $5=$ "much worse now than one year ago." Improved: Percentage of patients improved from baseline.

CI, confidence interval; SRM, standardized Response Mean effect size (small: $0.20-0.49$, medium: 0.50-0.79, large: $=0.80$ ); $S F-36$, Short Form-3b Health Survey.

sensitivity analyses (Table 4). The first sensitivity analysis (SA1) concerned dropout bias. The main analysis had comprised all patients with evaluable data at baseline and 12-month follow-up. In SA1, missing values after 12 months were replaced with the last value carried forward, reducing the average $0-12$-month improvement by $6 \%$ $(2.63 \rightarrow 2.46$ points). SA2 concerned the effect of AM adjunctive therapies: The sample was restricted to patients using neither eurythmy nor art therapy in the first 6 study months, reducing the improvement by $4 \%(2.63 \rightarrow 2.52$ points).
SA3 concerned spontaneous improvement, which was assumed to be improbable in patients with disease duration $\geq 12$ months: The sample was restricted to patients with disease duration of $\geq 12$ months prior to study enrollment, reducing the improvement by $4 \%(2.63 \rightarrow 2.53$ points $)$.

Combining SA1 + SA2 + SA3, the improvement was reduced by a total of $10 \%$.

SA4 concerned the effects of relevant non-AM adjunctive therapies, and was performed on patients with a main diagnosis of musculoskeletal or mental diseases or headache disorders. Restricting this sample to patients not using di-

Table 4. Symptom Score 0-12 Months: Sensitivity Analysis (SA)

\begin{tabular}{|c|c|c|c|c|c|}
\hline & & 0 months & 12 months & 0-12-month difference & \\
\hline Item & $N$ & Mean $\pm S D$ & Mean $\pm S D$ & Mean $(95 \%$ CI) & $\mathrm{p}$ value \\
\hline $\begin{array}{l}\text { Main analysis: patients enrolled after } 1 / 1 / 1999^{a} \\
\text { with evaluable data at } 0 \text { and } 12 \text { months }\end{array}$ & 59 & $5.76 \pm 1.81$ & $3.13 \pm 2.20$ & $2.63(2.02-3.23)$ & $p<0.001$ \\
\hline SA1: last value carried forward & 71 & $5.72 \pm 1.78$ & $3.27 \pm 2.31$ & $2.46(1.91-3.00)$ & $p<0.001$ \\
\hline $\begin{array}{l}\text { SA2: patients not using eurythmy or art therapy } \\
\text { in months } 0-6\end{array}$ & 49 & $5.64 \pm 1.85$ & $3.12 \pm 2.05$ & $2.52(1.87-3.17)$ & $p<0.001$ \\
\hline $\begin{array}{l}\text { SA3: patients with disease duration } \geq 12 \\
\text { months at study enrollment }\end{array}$ & 39 & $5.84 \pm 1.80$ & $3.31 \pm 1.99$ & $2.53(1.78-3.29)$ & $p<0.001$ \\
\hline $\mathrm{SA} 1+\mathrm{SA} 2+\mathrm{SA} 3$ & 35 & $5.62 \pm 1.71$ & $3.24 \pm 1.62$ & $2.38(1.64-3.13)$ & $p<0.001$ \\
\hline \multicolumn{6}{|l|}{$\begin{array}{l}\text { Patients with main diagnosis of } \\
\text { musculoskeletal or mental diseases, } \\
\text { or headache disorders }\end{array}$} \\
\hline Main analysis & 39 & $5.75 \pm 1.95$ & $3.09 \pm 2.27$ & $2.66(1.95-3.37)$ & $p<0.001$ \\
\hline $\begin{array}{l}\text { SA4: patients not using diagnosis-related } \\
\text { adjunctive therapies (see text) in months } 0-6\end{array}$ & 17 & $5.15 \pm 1.45$ & $2.41 \pm 1.93$ & $2.74(1.87-3.61)$ & $p<0.001$ \\
\hline
\end{tabular}

aSymptom Score was not documented in patients enrolled before 1/1/1999.

$\mathrm{CI}$, confidence interval. 
agnosis-related adjunctive therapies during the first 6 study months (Table 2), the average Symptom Score improvement was increased by $3 \%(2.66 \rightarrow 2.74$ points $)$.

\section{Other outcomes}

Therapy ratings: At 6-month follow-up, patients' average therapy outcome rating (from 0 "no help at all" to 10 "helped very well") was $7.50 \pm 2.34$; patient satisfaction with therapy (from 0 "very dissatisfied" to 10 "very satisfied") was $8.18 \pm 2.08$. Patients' RMT effectiveness rating was positive ("very effective" or "effective") in $83 \%(57 / 69)$ of patients, and negative ("less effective," "ineffective," or "not evaluable") in 17\%. Physicians' effectiveness rating was positive in $77 \%$ (53/69) and negative in 23\%. Ratings of therapy outcome, satisfaction, and effectiveness did not differ significantly between adults and children, or between 6- and 12-month follow-ups.

Adverse reactions during the first 24 study months: Adverse reactions to RMT occurred in four (5\%) patients: (1) mild cardiac palpitations; (2) moderate arterial hypotension; (3) moderate pain and vertigo; and (4) moderate (patient report)/severe (physician report) symptom aggravation. Patients 3 and 4 stopped RMT because of adverse reactions. No adverse reactions to AM eurythmy or art therapy occurred. Adverse reactions to AM medications occurred in $6 \%$ (4/70 users); adverse reactions to non-AM medication occurred in $11 \%(8 / 74$ users $)(p=0.369)$.

Serious adverse events. One patient died of breast cancer 22 months after study enrollment; this event had no relation to any therapy or medication.

\section{DISCUSSION}

This is the first study focusing on RMT as primary therapy. We aimed to obtain information on RMT under routine conditions in Germany and studied clinical outcomes in outpatients referred to RMT for chronic diseases. The study was conducted in conjunction with a health insurance program providing RMT regardless of diagnosis. For this reason, and because the range and frequency of indications for RMT in outpatient care were largely unknown prior to the study, we included patients of all ages with all diagnoses. The most frequent indications were musculoskeletal and mental disorders. After RMT, substantial improvements of disease symptoms and quality of life were observed. Improvements occurred during the first 6 months and were maintained during the 4-year follow-up.

Strengths of this study include a long follow-up period, high follow-up rates, and the participation of $21 \%$ of all certified RMT therapists in Germany. Participants resembled all eligible therapists with respect to sociodemographic char- acteristics. These features suggest that the study to a high degree mirrors contemporary RMT practice.

Nevertheless, because the study had a long recruitment period, the participating physicians were not able to screen and include all patients referred to RMT. It was estimated that physicians enrolled $15 \%$ of such patients. Selection bias could be present if physicians would preferentially screen and enroll patients for whom a particularly positive outcome was expected. In this case, one would expect the degree of selection ( $=$ the proportion of referred versus enrolled patients) to correlate positively with clinical outcomes. That was not the case, the correlation was almost zero (0.10) and not significant. This analysis does not suggest that physicians' screening of patients referred to RMT was affected by selection bias.

Because 13 clinical outcomes were analyzed, the issue of multiple hypothesis-testing arises. ${ }^{10}$ However, all analyzed 0-12-month comparisons of clinical outcomes showed significant improvements (Table 3), and 11 of these 13 comparisons had $p$ values $\leq 0.005$.

Because patients with all diagnoses were included, our study offers a comprehensive picture of RMT practice. On the other hand, it was not feasible to have disease-specific outcomes for all diagnoses included. Nonetheless, this study is part of a larger AM evaluation project that included disease-specific outcomes for major disease groups. ${ }^{12,13}$

A limitation of the study is the absence of a comparison group receiving another treatment or no therapy. For the observed improvements, one has to consider several other causes apart from RMT. Therefore, sensitivity analyses were conducted in regard to dropout bias, adjunctive AM eurythmy or art therapy, and spontaneous improvement. According to the analyses, these three factors can together explain maximum $10 \%$ of the average $0-12$-month improvement. Notably, this analysis does not exclude regression to the mean caused by symptom fluctuation with preferential self-selection to therapy and study inclusion at symptom peaks. Another factor, non-AM adjunctive therapies, cannot explain the improvement, because the improvement was similar in patients not using such therapies (analyzed in patients with musculoskeletal or mental disease or headache disorders, $69 \%$ of the study sample). Other possible confounders are AM medication (used by three-fourths of patients), observation bias, and psychologic factors such as patient expectations. However, because RMT was evaluated as a therapy package, the question of specific therapy effects versus nonspecific effects (placebo effects, context effects, patient expectations, etc.) was not an issue of the present analysis.

Because RMT was to be evaluated under routine conditions, therapy was administered at the discretion of the RMT therapists and not according to a standardized protocol. This raises the question of whether study interventions would be replicable in future studies. However, RMT therapists worldwide are taught the same set of RMT techniques ac- 
cording to a highly standardized curriculum. Therefore, relevant therapy differences across settings would not be expected. Moreover, in this study, any local therapy differences would probably be offset by the relatively large number of participating RMT therapists.

Previous studies have evaluated AM therapy including RMT in inpatient settings and found improved quality of life in breast cancer patients; ${ }^{5}$ improved coping with chronic musculoskeletal pain; ${ }^{6}$ high anorexia nervosa cure rates; ${ }^{3}$ and reduced pain, reduced medication use, and earlier return to work in lumbar disc disease. ${ }^{3}$ In accordance with these findings, our primary care study of patients with predominantly musculoskeletal and mental diseases demonstrated longstanding clinically relevant improvements in disease symptoms and quality of life. At 12-month follow-up, two-thirds of the patients had a clinically relevant symptom improvement of at least $30 \%$ of their baseline score. Altogether, the positive outcome of RMT in this study is encouraging.

\section{CONCLUSIONS}

In this first study focusing on RMT, patients treated with RMT had substantial long-term reduction of chronic disease symptoms and improvement of quality of life.

\section{ACKNOWLEDGMENTS}

This study was funded by the Software-AG Stiftung and the Innungskrankenkasse Hamburg.

\section{REFERENCES}

1. Steiner R, Wegman I. Extending practical medicine. Fundamental principles based on the science of the spirit. GA 27. Bristol: Rudolf Steiner Press, 2000, 144 p.

2. Ritchie J, Wilkinson J, Gantley M, et al. A model of integrated primary care: Anthroposophic medicine. London. Department of General Practice and Primary Care, St. Bartholomew's and the Royal London School of Medicine and Dentistry, Queen Mary University of London, 2001.
3. Kienle GS, Kiene H, Albonico HU. Anthroposophic medicine: Effectiveness, utility, costs, safety. Stuttgart, New York: Schattauer Verlag, 2006, 336 p.

4. Hauschka-Stavenhagen M. Rhythmical Massage as Indicated by Dr. Ita Wegman. Spring Valley, NY: Mercury Press, 1990, 138 p.

5. Carlsson M, Arman M, Backman M, et al. Evaluation of quality of life/life satisfaction in women with breast cancer in complementary and conventional care. Acta Oncol 2004;43:27-34.

6. Flatters U, Arman M, Ekenbäck K. Pain rehabilitation in the Vidar Clinic: Report of a project at the Vidar Clinic in collaboration with Scania Partner AB and the Swedish Social Insurance Administration in Stockholm, 1988. Järna: Vidarkliniken, 1998, 24 p.

7. Hamre HJ, Becker-Witt C, Glockmann A, et al. Anthroposophic therapies in chronic disease: The Anthroposophic Medicine Outcomes Study (AMOS). Eur J Med Res 2004; 9:351-360.

8. Hamre HJ, Witt CM, Glockmann A, et al. Health costs in anthroposophic therapy users: A two-year prospective cohort study. BMC Health Serv Res 2006;6:doi:10.1186/1472-6963-6-65.

9. Bullinger M, Kirchberger I. SF-36 Health Survey, German Version: Handbook. Göttingen: Hogrefe-Verlag, 1998, 155 p.

10. Feise RJ. Do multiple outcome measures require $p$-value adjustment? BMC Med Res Methodol 2002;2:8.

11. Liang MH, Fossel AH, Larson MG. Comparisons of five health status instruments for orthopedic evaluation. Med Care 1990;28:632-642.

12. Hamre HJ, Witt CM, Glockmann A, et al. Anthroposophic therapy for chronic depression: A four-year prospective cohort study. BMC Psychiatry 2006;6:doi:10.1186/1471244X-6-57.

13. Hamre HJ, Witt CM, Glockmann A, et al. Anthroposophic vs. conventional therapy for chronic low back pain: A prospective non-randomised comparative study. Eur J Med Res 2007;12:1-9.

Address reprint requests to:

Harald J. Hamre, M.D.

Institute for Applied Epistemology and Medical Methodology

Abteilung für Klinische Forschung Böcklerstraße 5 D-79110 Freiburg, Germany

E-mail: harald.hamre@ifaemm.de 